\title{
On Comparison Meaningfulness of Aggregation Functions
}

\author{
Jean-Luc Marichal* Pierre Mathonet $^{\dagger}$ \\ Revised version, August 25, 1999
}

\begin{abstract}
This paper will give a description of all continuous functions which are comparison meaningful in the sense of measurement theory.
\end{abstract}

Keywords: Comparison meaningfulness; ordinal scales; aggregation functions; decision making.

\section{Introduction}

Let $\underline{x}=\left(x_{1}, \ldots, x_{n}\right)$ be a vector whose components are real numbers determined only up to the order. Then any $n$-tuple $\underline{x}^{\prime}=\left(x_{1}^{\prime}, \ldots, x_{n}^{\prime}\right)$ represents the same information as $\underline{x}$, provided there exists a continuous and strictly increasing function $\phi$ such that $x_{i}^{\prime}=\phi\left(x_{i}\right)$ for all $i=1, \ldots, n$. These values are thus given according to an ordinal scale. For example, the scale of air quality being used in a number of cities is an ordinal scale. It assigns a number 1 to unhealthy air, 2 to unsatisfactory air, 3 to acceptable air, 4 to good air, and 5 to excellent air. We could use the numbers $1,7,8,15,23$, as well, or the numbers 1.2 , $6.5,8.7,205.6,750$, or any other numbers that keep the order. An extensive study of such scales can be found in Roberts' book [10] on measurement theory.

In this paper we deal with the problem of aggregation of ordinal values. Suppose that $x_{1}, \ldots, x_{n} ; x_{1}^{\prime}, \ldots, x_{n}^{\prime}$ are numbers defined on the same ordinal scale and that, using an aggregation function $M$, we can compare two aggregated values

$$
M\left(x_{1}, \ldots, x_{n}\right)\left\{\begin{array}{l}
< \\
=
\end{array}\right\} M\left(x_{1}^{\prime}, \ldots, x_{n}^{\prime}\right) .
$$

It seems sensible to assume that we have

$$
M\left(\phi\left(x_{1}\right), \ldots, \phi\left(x_{n}\right)\right)\left\{\begin{array}{l}
< \\
=
\end{array}\right\} M\left(\phi\left(x_{1}^{\prime}\right), \ldots, \phi\left(x_{n}^{\prime}\right)\right)
$$

for any continuous and strictly increasing function $\phi$. Such an aggregation function $M$ is said to be comparison meaningful (see Orlov [7]).

In application, suppose that each of $n$ voters gives a complete ranking (a complete and transitive binary relation) on a set of candidates (or alternatives) $A=\{a, b, \ldots\}$. This ranking can be given by means of values defined on an ordinal scale (see Table 1 ).

\footnotetext{
*Jean-Luc Marichal is with the Department of Management, FEGSS, University of Liège, Boulevard du Rectorat 7 - B31, B-4000 Liège, Belgium, Email: jl.marichal[at]ulg.ac.be

${ }^{\dagger}$ Pierre Mathonet is with the Institute of Mathematics, University of Liège, Grande Traverse 12 - B37, Sart Tilman, B-4000 Liège, Belgium, Email: p.mathonet[at]ulg.ac.be
} 


\begin{tabular}{|c|cccc|c|}
\cline { 2 - 6 } \multicolumn{1}{c|}{} & Voter 1 & Voter 2 & $\cdots$ & Voter $n$ & Social ranking \\
\hline Candidate $a$ & $x_{1}^{a}$ & $x_{2}^{a}$ & $\cdots$ & $x_{n}^{a}$ & $M\left(x_{1}^{a}, \ldots, x_{n}^{a}\right)$ \\
Candidate $b$ & $x_{1}^{b}$ & $x_{2}^{b}$ & $\cdots$ & $x_{n}^{b}$ & $M\left(x_{1}^{b}, \ldots, x_{n}^{b}\right)$ \\
$\vdots$ & $\vdots$ & $\vdots$ & & $\vdots$ & $\vdots$ \\
\hline
\end{tabular}

Table 1: Voting procedure

We can aggregate the $n$ opinions to build a social ranking of the candidates. It is obvious that any function $M$ involved in such a procedure must be such that

$$
M\left(x_{1}, \ldots, x_{n}\right)\left\{\begin{array}{l}
< \\
=
\end{array}\right\} M\left(x_{1}^{\prime}, \ldots, x_{n}^{\prime}\right)
$$

implies

$$
M\left(\phi_{1}\left(x_{1}\right), \ldots, \phi_{n}\left(x_{n}\right)\right)\left\{\begin{array}{l}
< \\
=
\end{array}\right\} M\left(\phi_{1}\left(x_{1}^{\prime}\right), \ldots, \phi_{n}\left(x_{n}^{\prime}\right)\right)
$$

for any continuous and strictly increasing functions $\phi_{1}, \ldots, \phi_{n}$. Kim [4, Corollary 1.2] showed that such a function $M$, when continuous, either is a constant function or there exists an index $k \in\{1, \ldots, n\}$ and a continuous and strictly monotonic function $g$ such that

$$
M\left(x_{1}, \ldots, x_{n}\right)=g\left(x_{k}\right) .
$$

We thus observe that such a voting procedure leads to the presence of a dictator.

Instead of considering independent ordinal scales, we assume that all the values $x_{i}^{a}$ in the table are defined on the same ordinal scale (commensurability assumption). This means that the implication

$$
x_{i}^{a}\left\{\begin{array}{l}
< \\
=
\end{array}\right\} x_{j}^{b} \Rightarrow \phi_{i}\left(x_{i}^{a}\right)\left\{\begin{array}{l}
< \\
=
\end{array}\right\} \phi_{j}\left(x_{j}^{b}\right)
$$

holds for any pair of candidates $a, b$ and any pair of voters $i, j$. In this case, we can immediately see that $\phi_{1}=\cdots=\phi_{n}=\phi$ and that $M$ must be comparison meaningful.

In this paper we intend to give a description of the family of all continuous and comparison meaningful aggregation functions. In Section 4 , it is shown that such a function $M$ either is a constant function or there exists a non-empty family $\left\{T_{k}\right\}_{k=1}^{m}$ of non-empty subsets of $\{1, \ldots, n\}$ and a continuous and strictly monotonic function $g$ such that

$$
M\left(x_{1}, \ldots, x_{n}\right)=g\left(\bigvee_{k=1}^{m} \bigwedge_{i \in T_{k}} x_{i}\right)
$$

where $\wedge$ and $\vee$ denote the min and max operations, respectively.

As a preliminary, we investigate in Section 3 the case of idempotent functions, that is functions $M$ such that $M(x, \ldots, x)=x$. Note that the case of symmetric and idempotent functions has already been investigated by Marichal and Roubens [5] and in the more general framework of ordered sets by Ovchinnikov [8]. The associated results have been summarized in [3, Chap. 5].

Similar studies have been done for ratio scales, interval scales, and log-interval scales by Aczél and Roberts [1] and Aczél, Roberts, and Rosenbaum [2]. 


\section{Basic definitions}

First we want to define the concept of aggregation function. We assume that the numbers to be aggregated belong to any closed interval $[a, b]$ of the real line. Let $n$ denote any strictly positive integer.

Definition 2.1 An aggregation function defined on $[a, b]^{n}$ is a function $M:[a, b]^{n} \rightarrow \mathbb{R}$.

We consider a set of $n$ elements $N=\{1, \ldots, n\}$, which could be voters, criteria, or attributes in a decision making problem. $2^{N}$ indicates the power set of $N$, i.e., the set of all subsets in $N$.

In order to avoid an heavy notation, we introduce the following terminology:

- We let $\Phi$ denote the set of all continuous and strictly increasing functions $\phi:[a, b] \rightarrow$ $[a, b]$. If $\underline{x}=\left(x_{1}, \ldots, x_{n}\right) \in[a, b]^{n}$ then $\phi(\underline{x})$ means $\left(\phi\left(x_{1}\right), \ldots, \phi\left(x_{n}\right)\right)$.

- For any $T \subseteq N$, the characteristic vector of $T$ in $\{0,1\}^{n}$ is defined by

$$
e_{T}:=\left(x_{1}, \ldots, x_{n}\right) \in\{0,1\}^{n} \text { with } x_{i}=1 \Leftrightarrow i \in T \text {. }
$$

Then, for any aggregation function $M$ defined on $[a, b]^{n}$, we set

$$
M_{T}:=M\left(a e_{N \backslash T}+b e_{T}\right) .
$$

- Given a vector $\left(x_{1}, \ldots, x_{n}\right) \in[a, b]^{n}$, let $(\cdot)$ be the permutation on $N$ which arranges the elements of this vector by increasing values: that is, $x_{(1)} \leq \ldots \leq x_{(n)}$.

According to Orlov [7], we can define the comparison meaningfulness of aggregation functions as follows:

Definition 2.2 The aggregation function $M$ defined on $[a, b]^{n}$ is comparison meaningful $(\mathrm{CM})$ if, for any $\phi \in \Phi$ and any $\underline{x}, \underline{x}^{\prime} \in[a, b]^{n}$, we have

$$
M(\underline{x})\left\{\begin{array}{l}
< \\
=
\end{array}\right\} M\left(\underline{x}^{\prime}\right) \Rightarrow M(\phi(\underline{x}))\left\{\begin{array}{l}
< \\
=
\end{array}\right\} M\left(\phi\left(\underline{x}^{\prime}\right)\right) .
$$

\section{Case of idempotent functions}

Let us consider some aggregation properties often encountered in the literature.

Definition 3.1 The aggregation function $M$ defined on $[a, b]^{n}$ is

- continuous (Co) if $M$ is a continuous function on $[a, b]^{n}$.

- idempotent (Id) if $M(x, \ldots, x)=x$ for all $x \in[a, b]$.

- increasing (In) if $M$ is increasing in each argument, i.e. if, for any $\underline{x}, \underline{x}^{\prime} \in[a, b]^{n}$, we have

$$
x_{i} \leq x_{i}^{\prime} \forall i \in N \Rightarrow M(\underline{x}) \leq M\left(\underline{x}^{\prime}\right) .
$$

- symmetric (Sy) if $M$ is a symmetric function on $[a, b]^{n}$, i.e. if, for any permutation $\sigma$ on $N$ and any $\underline{x} \in[a, b]^{n}$, we have

$$
M\left(x_{1}, \ldots, x_{n}\right)=M\left(x_{\sigma(1)}, \ldots, x_{\sigma(n)}\right) .
$$


The properties mentioned in Definition 3.1 are rather natural. If we are searching for functions which do not present any chaotic reaction to a small change of the arguments, we confine ourselves to smooth functions, i.e., functions fulfilling (Co). The (Id) property clearly expresses the unanimity principle. (In) imposes that the functions present a nonnegative response to any increase of the arguments, and (Sy) leads us to neutral functions, i.e., independent of the labels.

In this section we describe the class of all the functions fulfilling (CM, Id, Co). We will see that those functions also fulfil (In). In particular, they are compensative, that is such that

$$
\min \underline{x} \leq M(\underline{x}) \leq \max \underline{x} \quad \forall \underline{x} \in[a, b]^{n} .
$$

The following lemma will be very useful as we continue.

Lemma 3.1 Let $M$ be an aggregation function defined on $[a, b]^{n}$ and satisfying $(C M, I d)$. If $M$ fulfils (In) or (Co) then

(i) $M(\phi(\underline{x}))=\phi(M(\underline{x}))$ for all $\phi \in \Phi$ and all $\underline{x} \in[a, b]^{n}$,

(ii) $M(\underline{x}) \in\left\{x_{1}, \ldots, x_{n}\right\}$ for all $\underline{x} \in[a, b]^{n}$.

Proof. This result was proved by Ovchinnikov [8] in the case of compensative functions fulfilling (CM, Id). Hence, we only have to prove that, under our assumptions, $M$ is compensative.

Assume first that $M$ fulfils (CM, Id, In). Then, for any $\underline{x} \in[a, b]^{n}$, we have

$$
\min \underline{x} \stackrel{(\mathrm{Id})}{=} M(\min \underline{x}, \ldots, \min \underline{x}) \stackrel{(\mathrm{In})}{\leq} M(\underline{x}) \stackrel{(\mathrm{In})}{\leq} M(\max \underline{x}, \ldots, \max \underline{x}) \stackrel{(\mathrm{Id})}{=} \max \underline{x} .
$$

Assume now that $M$ fulfils (CM, Id, Co). Consider for example the case of max. Let $\underline{x} \in] a, b\left[^{n}\right.$ and suppose that $M(\underline{x})>\max \underline{x}$. Then there exists $\left.\theta \in\right] a, b[$ such that $M(\underline{x})>\theta>\max \underline{x}$. For any $m \in \mathbb{N}_{0}$, we construct the function $\phi_{m} \in \Phi$ by linear interpolation of the points $(a, a),(\theta, \theta),(b, b)$, and $\left(x_{i}, \max \underline{x}-\left(\max \underline{x}-x_{i}\right) / m\right)$ for all $i \in N$. We have

$$
M(\underline{x})>\theta \stackrel{(\mathrm{Id})}{=} M(\theta, \ldots, \theta) .
$$

Using (CM), we have, for any $m \in \mathbb{N}_{0}$,

$$
M\left(\phi_{m}(\underline{x})\right)>M\left(\phi_{m}(\theta), \ldots, \phi_{m}(\theta)\right)=M(\theta, \ldots, \theta)=\theta .
$$

However, we have

$$
\lim _{m \rightarrow \infty} M\left(\phi_{m}(\underline{x})\right) \stackrel{(\mathrm{Co})}{=} M\left(\lim _{m \rightarrow \infty} \phi_{m}(\underline{x})\right)=M(\max \underline{x}, \ldots, \max \underline{x})=\max \underline{x} .
$$

Consequently, we have $\max \underline{x} \geq \theta$, a contradiction.

The result is then true for any $\underline{x} \in] a, b\left[^{n}\right.$. By continuity of $M$, it is also true on $[a, b]^{n}$.

Now, let us introduce the functions which play a central role in this paper: the Boolean max-min functions. They are of course to be compared with the classical increasing Boolean functions.

Definition 3.2 For any set function $c: 2^{N} \rightarrow\{0,1\}$ such that $c_{\emptyset}=0$ and $\bigvee_{T \subseteq N} c_{T}=1$, the Boolean max-min function $\mathrm{B}_{c}^{\vee \wedge}$ defined on $[a, b]^{n}$ and associated to $c$ is defined by

$$
\mathrm{B}_{c}^{\vee \wedge}(\underline{x})=\bigvee_{\substack{T \subseteq N \\ c_{T}=1}} \bigwedge_{i \in T} x_{i}, \quad \underline{x} \in[a, b]^{n} .
$$


Using classical distributivity of $\wedge$ and $\vee$, we can see that any Boolean max-min function can also be put in the form

$$
\bigwedge_{\substack{T \subseteq N \\ d_{T}=0}} \bigvee_{i \in T} x_{i}
$$

with an appropriate set function $d: 2^{N} \rightarrow\{0,1\}$ such that $d_{\emptyset}=1$ and $\bigwedge_{T \subseteq N} d_{T}=0$ (see [6] for more details).

Now, let us turn to the first characterization, as mentioned in the beginning of this section.

Theorem 3.1 Let $M$ be any aggregation function defined on $[a, b]^{n}$. Then the following three assertions are equivalent:

(i) $M$ fulfils (CM, Id, Co)

(ii) $M$ fulfils (CM, Id, In)

(iii) There exists a set function $c$ such that $M=\mathrm{B}_{c}^{\vee \wedge}$.

Proof. $(i) \Rightarrow($ ii $)$ Consider $\underline{x}=\left(x_{1}, \ldots, x_{n}\right) \in[a, b]^{n}$ reordered as $x_{(1)} \leq \ldots \leq x_{(n)}$ and set $x_{(0)}:=a, x_{(n+1)}:=b$. Let $k \in N$ and consider the function $f_{k}:[a, b] \rightarrow \mathbb{R}$ defined by

$$
f_{k}(t):=M\left(\underline{x}+\left(t-x_{k}\right) e_{k}\right), \quad t \in[a, b] .
$$

We will show that $f_{k}$ is increasing on $[a, b]$. Let $i \in\{0,1, \ldots, n\}$ such that $x_{(i)}<x_{(i+1)}$. Then $f_{k}$ is increasing on $] x_{(i)}, x_{(i+1)}$ [. Indeed, if $\left.t_{1}, t_{2} \in\right] x_{(i)}, x_{(i+1)}\left[, t_{2}>t_{1}\right.$, then there exists $\phi \in \Phi$ such that $\phi\left(t_{1}\right)=t_{2}, \phi\left(x_{(j)}\right)=x_{(j)}$ for all $j \in\{0,1, \ldots, n+1\}$ and $\phi(t) \geq t$ for all $t \in[a, b]$. Then, by Lemma 3.1, we have

$$
f_{k}\left(t_{2}\right)=f_{k}\left(\phi\left(t_{1}\right)\right)=\phi\left(f_{k}\left(t_{1}\right)\right) \geq f_{k}\left(t_{1}\right) .
$$

Finally, by (Co), $f_{k}$ is increasing on $[a, b]$.

(ii) $\Rightarrow($ iii $)$ By Lemma 3.1, we have $M_{T} \in\{a, b\}$ for all $T \subseteq N$, where $M_{T}$ is defined by (1). Moreover, for all $x \in[a, b]$ and all $T \subseteq N$, we have

$$
\begin{aligned}
M\left(a e_{N \backslash T}+x e_{T}\right) & =M_{T} \wedge x \\
M\left(x e_{T}+b e_{N \backslash T}\right) & =M_{N \backslash T} \vee x
\end{aligned}
$$

Indeed, using Lemma 3.1 with $\phi(t)=\frac{x-a}{b-a}(t-a)+a$, we have

$$
M\left(a e_{N \backslash T}+x e_{T}\right)=M\left(\phi(a) e_{N \backslash T}+\phi(b) e_{T}\right)=\phi\left(M_{T}\right),
$$

which proves (2). Similarly, using Lemma 3.1 with $\phi(t)=\frac{b-x}{b-a}(t-a)+x$, we have

$$
M\left(x e_{T}+b e_{N \backslash T}\right)=M\left(\phi(a) e_{T}+\phi(b) e_{N \backslash T}\right)=\phi\left(M_{N \backslash T}\right),
$$

which proves $(3)$.

Let $\underline{x} \in[a, b]^{n}$. On the one hand, for all $T \subseteq N$, we have

$$
M(\underline{x}) \stackrel{(\text { In })}{\geq} M\left[a e_{N \backslash T}+\left(\bigwedge_{i \in T} x_{i}\right) e_{T}\right] \stackrel{(2)}{=} M_{T} \wedge\left(\bigwedge_{i \in T} x_{i}\right)
$$

and thus

$$
M(\underline{x}) \geq \bigvee_{T \subseteq N}\left[M_{T} \wedge\left(\bigwedge_{i \in T} x_{i}\right)\right]
$$


On the other hand, let $T^{*} \subseteq N$ such that $M_{T^{*}} \wedge\left(\bigwedge_{i \in T^{*}} x_{i}\right)$ is maximum and set

$$
J:=\left\{j \in N \mid x_{j} \leq M_{T^{*}} \wedge\left(\bigwedge_{i \in T^{*}} x_{i}\right)\right\} .
$$

We should have $J \neq \emptyset$. Indeed, if $x_{j}>M_{T^{*}} \wedge\left(\bigwedge_{i \in T^{*}} x_{i}\right)$ for all $j \in N$, we have, since $M_{N}=b$,

$$
M_{N} \wedge\left(\bigwedge_{i \in N} x_{i}\right)=\bigwedge_{i \in N} x_{i}>M_{T^{*}} \wedge\left(\bigwedge_{i \in T^{*}} x_{i}\right)
$$

which contradicts the definition of $T^{*}$. Moreover, we should have $M_{N \backslash J}=a$. Indeed, this is trivial if $N \backslash J=\emptyset$; otherwise, if $N \backslash J \neq \emptyset$ and $M_{N \backslash J}=b$ then we have, by definition of $J$,

$$
M_{N \backslash J} \wedge\left(\bigwedge_{i \in N \backslash J} x_{i}\right)=\bigwedge_{i \in N \backslash J} x_{i}>M_{T^{*}} \wedge\left(\bigwedge_{i \in T^{*}} x_{i}\right),
$$

a contradiction. Finally, we have,

$$
M(\underline{x}) \stackrel{(\mathrm{In})}{\leq} M\left[\left(M_{T^{*}} \wedge\left(\bigwedge_{i \in T^{*}} x_{i}\right)\right) e_{J}+b e_{N \backslash J}\right] \stackrel{(3)}{=} M_{T^{*}} \wedge\left(\bigwedge_{i \in T^{*}} x_{i}\right),
$$

that is

$$
M(\underline{x}) \leq \bigvee_{T \subseteq N}\left[M_{T} \wedge\left(\bigwedge_{i \in T} x_{i}\right)\right]
$$

Now, setting $c_{T}:=\left(M_{T}-a\right) /(b-a)$ for all $T \subseteq N$, we have $c_{\emptyset}=0, c_{N}=1$ and, by (4)-(5), we have

$$
M(\underline{x})=\bigvee_{T \subseteq N}\left[M_{T} \wedge\left(\bigwedge_{i \in T} x_{i}\right)\right]=\bigvee_{\substack{T \subseteq N \\ M_{T}=b}} \bigwedge_{i \in T} x_{i}=\bigvee_{\substack{T \subseteq N \\ c_{T}=1}} \bigwedge_{i \in T} x_{i}
$$

$($ iii $) \Rightarrow(i)$ Of course, the Boolean max-min functions satisfy (Id, Co). They also satisfy $(\mathrm{CM})$; indeed, we have $\phi(x \vee y)=\phi(x) \vee \phi(y)$ and $\phi(x \wedge y)=\phi(x) \wedge \phi(y)$ for all $\phi \in \Phi$ and all $x, y \in[a, b]$, which is sufficient.

In order to study the particular case of symmetric functions, we need to introduce the so-called order statistics (cf. van der Waerden [11, Sect. 17]).

Definition 3.3 For any $k \in N$, the order statistic function $\operatorname{OS}_{k}$ defined on $[a, b]^{n}$ and associated to the $k$-th argument is defined by $\operatorname{OS}_{k}(\underline{x})=x_{(k)}$ for all $\underline{x} \in[a, b]^{n}$.

Ovchinnikov [8, Sect. 7] proved that for any $\underline{x} \in[a, b]^{n}$ and any $k \in N$, we have

$$
x_{(k)}=\bigvee_{1 \leq i_{1}<\ldots<i_{n-k+1} \leq n}\left(x_{i_{1}} \wedge \ldots \wedge x_{i_{n-k+1}}\right) .
$$

Therefore, the order statistic $\mathrm{OS}_{k}$ is a Boolean max-min function such that $c_{T}=1$ if and only if $|T|=n-k+1$. The following result shows that the order statistics are the symmetric Boolean max-min functions.

Corollary 3.1 Let $M$ be any aggregation function defined on $[a, b]^{n}$. Then the following three assertions are equivalent:

(i) $M$ fulfils (CM, Id, Co, Sy)

(ii) $M$ fulfils (CM, Id, In, Sy)

(iii) There exists $k \in N$ such that $M=\mathrm{OS}_{k}$. 
Proof. $(i) \Leftrightarrow(i i)$. See Theorem 3.1.

(i)\&(ii) $\Rightarrow$ (iii) See Marichal and Roubens [5] and Ovchinnikov [8].

(iii) $\Rightarrow($ ii) Trivial.

Remark. If we replace $\Phi$ by the automorphism group of $[a, b]$, that is, the set of all continuous and strictly increasing functions $\phi:[a, b] \rightarrow[a, b]$ with boundary conditions $\phi(a)=a, \phi(b)=b$, then the (CM) property is weakened and the equivalence between $(i)$ and $(i i)$ in Theorem 3.1 does not hold anymore. Indeed, if $\left(\mathrm{CM}^{\prime}\right)$ represents $(\mathrm{CM})$ with the new $\Phi$, then we immediately see that the non-continuous function

$$
M(\underline{x})= \begin{cases}b, & \text { if } \max _{i} x_{i}=b, \\ \min _{i} x_{i}, & \text { else }\end{cases}
$$

fulfils (CM', Id, In). The same observation can be made for Corollary 3.1.

In the more general case where $M$ is defined on $X^{n}, X$ being a doubly homogeneous linear order, and where $\Phi$ is the automorphism group of $X$, the equivalence between $(i)$ and (iii) in Theorem 3.1 was independently established in [9] using a rather different approach.

\section{Main result}

Before turning to the main characterization, we need to consider some technical lemmas. Moreover, for any partition $(R, S, T)$ of $N$, we define the function $M_{(R, S, T)}:[a, b] \rightarrow \mathbb{R}$ by

$$
M_{(R, S, T)}(t):=M\left(a e_{R}+t e_{S}+b e_{T}\right), \quad t \in[a, b] .
$$

Lemma 4.1 Let $M$ be any aggregation function defined on $[a, b]^{n}$ and satisfying $(C M$, Co). Then, for any partition $(R, S, T)$ of $N$, the function $M_{(R, S, T)}$ is constant or strictly monotonic.

Proof. Let $\left.x_{0}, y_{0} \in\right] a, b\left[\right.$ such that $x_{0}<y_{0}$ and suppose $M_{(R, S, T)}\left(x_{0}\right)<M_{(R, S, T)}\left(y_{0}\right)$ (resp. $>,=)$. Let $x, y \in] a, b[$ such that $x<y$. There exists $\phi \in \Phi$ such that $\phi(a)=a, \phi(b)=$ $b, \phi\left(x_{0}\right)=x$ and $\phi\left(y_{0}\right)=y$. By $(\mathrm{CM})$, we have $M_{(R, S, T)}(x)<M_{(R, S, T)}(y)(\operatorname{resp} .>,=)$. Finally, by (Co), $M_{(R, S, T)}$ is strictly increasing (resp. strictly decreasing, constant) on $[a, b]$.

Lemma 4.2 Let $M$ be any aggregation function defined on $[a, b]^{n}$ and satisfying $(C M, C o)$. Then, there exist $T, T^{\prime} \subseteq N$ such that $M_{T} \leq M(\underline{x}) \leq M_{T^{\prime}}$ for all $\underline{x} \in[a, b]^{n}$.

Proof. Let us consider the case of the lower bound. The other one can be dealt with similarly. By $(\mathrm{Co})$, there exists $\underline{x}^{*} \in[a, b]^{n}$ such that $M\left(\underline{x}^{*}\right) \leq M(\underline{x})$ for all $\underline{x} \in[a, b]^{n}$. Let

$$
C:=\left\{x_{i}^{*} \mid x_{i}^{*} \in\right] a, b[\} .
$$

If $C=\emptyset$ then we can conclude immediately. Else, let

$$
K:=\left\{k \in N \mid x_{k}^{*}=\min C\right\} \neq \emptyset .
$$

Choosing $k \in K$, we have

$$
\left.M\left(\underline{x}^{*}\right)=M\left(\underline{x}^{*}+\left(x-x_{k}^{*}\right) e_{K}\right) \quad \forall x \in\right] a, x_{k}^{*}[\cdot
$$


Indeed, suppose that there exists $x \in] a, x_{k}^{*}\left[\right.$ such that $M\left(\underline{x}^{*}\right)<M\left(\underline{x}^{*}+\left(x-x_{k}^{*}\right) e_{K}\right)$ and consider $\phi \in \Phi$ such that $\phi(a)=a, \phi(b)=b, \phi(x)=x_{k}^{*}$ and $\phi\left(x_{i}^{*}\right)=x_{i}^{*}$ for all $i \in N \backslash K$. By $(\mathrm{CM})$, we have $M\left(\phi\left(\underline{x}^{*}\right)\right)<M\left(\underline{x}^{*}\right)$, a contradiction.

By (Co), we have

$$
M\left(\underline{x}^{*}\right)=M\left(\underline{x}^{*}+\left(a-x_{k}^{*}\right) e_{K}\right) .
$$

We can iterate this process until we obtain $M\left(\underline{x}^{*}\right)=M_{T}$ with $T=\left\{i \in N \mid x_{i}^{*}=b\right\}$.

Lemma 4.3 Let $M$ be any aggregation function defined on $[a, b]^{n}$ and satisfying $(C M, C o)$. Then, we have $M_{\emptyset} \leq M(\underline{x}) \leq M_{N}$ or $M_{N} \leq M(\underline{x}) \leq M_{\emptyset}$ for all $\underline{x} \in[a, b]^{n}$.

Proof. Let us consider the case of the lower bound. The other one can be dealt with similarly. By Lemma 4.2, there exists $T \subseteq N$ such that $M_{T} \leq M(\underline{x})$ for all $\underline{x} \in[a, b]$. By Lemma 4.1, we have three mutually exclusive cases:

- If $M_{(\emptyset, N, \emptyset)}$ is strictly increasing then $M_{\emptyset}<M_{N}$. Suppose $M_{T}<M_{\emptyset}$. By Lemma 4.1, $M_{(\emptyset, N \backslash T, T)}$ is strictly increasing since

$$
M_{(\emptyset, N \backslash T, T)}(a)=M_{T}<M_{N}=M_{(\emptyset, N \backslash T, T)}(b) .
$$

By (Co), there exists $r \in] a, b\left[\right.$ such that $M_{\emptyset}=M_{(\emptyset, N \backslash T, T)}(r)$. Then, there exists $\phi_{i} \in \Phi$, such that $\phi_{i}(a)=a, \phi_{i}(b)=b$ and $\phi_{i}(r)=b-(b-r) / i$ for all $i \in \mathbb{N}_{0}$. By (CM), we have

$$
M_{\emptyset}=M_{(\emptyset, N \backslash T, T)}\left(\phi_{i}(r)\right), \quad i \in \mathbb{N}_{0},
$$

and by $(\mathrm{Co}), M_{\emptyset}=M_{N}$, a contradiction.

- If $M_{(\emptyset, N, \emptyset)}$ is strictly decreasing then $M_{N}<M_{\emptyset}$. Suppose $M_{T}<M_{N}$. By Lemma 4.1, $M_{(N \backslash T, T, \emptyset)}$ is strictly decreasing since

$$
M_{(N \backslash T, T, \emptyset)}(a)=M_{\emptyset}>M_{T}=M_{(N \backslash T, T, \emptyset)}(b) .
$$

We can conclude as in the previous case.

- If $M_{(\emptyset, N, \emptyset)}$ is constant then $M_{\emptyset}=M_{N}$. Suppose $M_{T}<M_{\emptyset}$. By Lemma $4.1, M_{(N \backslash T, T, \emptyset)}$ is strictly decreasing and $M_{(\emptyset, N \backslash T, T)}$ is strictly increasing. Taking $\left.r \in\right] a, b[$, we have

$$
M_{T}=M_{(N \backslash T, T, \emptyset)}(b)<M_{(N \backslash T, T, \emptyset)}(r)<M_{(N \backslash T, T, \emptyset)}(a)=M_{\emptyset}=M_{N} .
$$

Hence, by (Co), there exists $s \in] a, b[$ such that

$$
M_{(N \backslash T, T, \emptyset)}(r)=M_{(\emptyset, N \backslash T, T)}(s) .
$$

Consider $\phi_{i} \in \Phi$ such that $\phi_{i}(a)=a, \phi_{i}(b)=b, \phi_{i}(r)=b-(b-r) / i$ and $\phi_{i}(s)=$ $b-(b-s) / i$ for all $i \in \mathbb{N}_{0}$. By $(\mathrm{CM})$ and $(\mathrm{Co})$, we have $M_{T}=M_{N}$, a contradiction.

Theorem 4.1 The aggregation function $M$ defined on $[a, b]^{n}$ fulfils $(C M, C o)$ if and only if

- either $M$ is constant, 
- or there exist a set function $c$ and a continuous strictly monotonic function $g:[a, b] \rightarrow$ $\mathbb{R}$ such that $M=g \circ \mathrm{B}_{c}^{\vee \wedge}$.

Proof. (Sufficiency) Easy.

(Necessity) By Lemma 4.1, $M_{(\emptyset, N, \emptyset)}$ is constant or strictly monotonic. If $M_{(\emptyset, N, \emptyset)}$ is constant then, by Lemma 4.3 , so is $M$. In the other case, the function $g:=M_{(\emptyset, N, \emptyset)}$ is a continuous bijection from $[a, b]$ onto $\left[M_{\emptyset} \wedge M_{N}, M_{\emptyset} \vee M_{N}\right]$. By Lemma 4.3, $M^{\prime}:=g^{-1} \circ M$ is well defined. Moreover, we can readily see that $M^{\prime}$ is an aggregation function defined on $[a, b]^{n}$ and satisfying (CM, Co). It also fulfils (Id) since

$$
M^{\prime}(x, \ldots, x)=g^{-1}(M(x, \ldots, x))=g^{-1}\left(M_{(\emptyset, N, \emptyset)}(x)\right)=x, \quad x \in[a, b] .
$$

Then, Theorem 3.1 enables us to conclude.

In the symmetric case, we have the following result, which is a straightforward consequence of Theorem 4.1 and Corollary 3.1.

Corollary 4.1 The aggregation function $M$ defined on $[a, b]^{n}$ fulfils (CM, Co, Sy) if and only if

- either $M$ is constant,

- or there exist $k \in N$ and a continuous strictly monotonic function $g:[a, b] \rightarrow \mathbb{R}$ such that $M=g \circ \mathrm{OS}_{k}$.

\section{Conclusions}

We have given a description of the class of all continuous and comparison meaningful aggregation functions. This generalizes some of the results obtained by Marichal and Roubens [5] and Ovchinnikov [8] about the order statistics.

\section{Acknowledgements}

The authors are indebted to Sergei Ovchinnikov for his useful comments. Jean-Luc Marichal gratefully acknowledges partial support by NATO (grant CRG 931531).

\section{References}

[1] J. Aczél and F.S. Roberts, On the possible merging functions, Math. Social Sciences 17 (1989) 205-243.

[2] J. Aczél, F.S. Roberts, and Z. Rosenbaum, On scientific laws without dimensional constants, J. of Math. Analysis and Appl. 119 (1986) 389-416.

[3] J. Fodor and M. Roubens, Fuzzy preference modelling and multicriteria decision support, (Kluwer, Dordrecht, 1994).

[4] S.-R. Kim, On the possible scientific laws, Mathematical Social Sciences 20 (1990) $19-36$. 
[5] J.-L. Marichal and M. Roubens, Characterization of some stable aggregation functions, in: Proc. Intern. Conf. on Industrial Engineering and Production Management, Mons, Belgium, June 2-4, 1993, pp. 187-196.

[6] J.-L. Marichal, On Sugeno integral as an aggregation function, Fuzzy Sets and Systems, to appear.

[7] A. Orlov, The connection between mean quantities and admissible transformations, Math. Notes 30 (1981) 774-778.

[8] S. Ovchinnikov, Means on ordered sets, Mathematical Social Sciences 32 (1996) 39-56.

[9] S. Ovchinnikov, Invariant functions on simple orders, ORDER 14 (1998) 365-371.

[10] F.S. Roberts, Measurement Theory with Applications to Decision-making, Utility and the Social Sciences, (Addison-Wesley Pub., Reading, MA, 1979).

[11] B.L. van der Waerden, Mathematical statistics, (Springer-Verlag, Berlin, 1969). 